\title{
Multi image stitching with cylindrical surface base on local feature matching for solving the distortion problem
}

\author{
Natthavut Vong a nun ${ }^{\mathrm{a}^{*}}$ and Chatklaw Jareonpon ${ }^{\mathrm{a}}$ \\ ${ }^{a}$ Mahasarakham University, Kantarawichai District, Maha Sarakham 44150, Thailand \\ *Corresponding Author: mikarukinji@gmail.com
}

\begin{abstract}
This paper presents a method to solve the problem encountered in wide view panorama image stitching. The stitching problem is appearing on the registration process using projective transformations model (homography) that will make the distort image severely, increasingly, and continually. In this paper, all images are transformed into the cylindrical surface instead of rectangle surface, then computed the translation of relation from image to find the placement. Experimental result using cylindrical surface shows that image is not enlarged and distorted by cylinder surface transformation.
\end{abstract}

Keywords: stitching image, cylindrical panorama, image transformation.

\section{Introduction}

Panorama image is a wide view angle than common image. Typically, a panorama image will be used for the landscape, and it is able to represent more realistic than the common image, because of the wide range of scene. The advantage of the panorama is a beautiful scene from surrounding such as travel photography, landscape and natural photography, and is also used for various medias. In addition, panorama images used for various applications or scientific research such as creating 3D virtual reality simulation, 3D medical diagnosis ${ }^{(1-3)}$.

The Algorithm used for merge multiple images with overlapping area is a well-known stitching image algorithm. Moreover, this technique is able to find the relation of image such as image segmentation ${ }^{(4)}$, and moving object detection $^{(5)}$. The stitching image algorithm is simple. Firstly, the features are extracted from the images and computes the matching feature using the Euclidian distance then the result is the pair of points with relation or similarity called corresponding point. The output from the previous process shows the overlap area of the images. In generally, the homography is computed from the estimated geometric transform and adjusts the image into the same perspective called warping images. After that, the image with warping set to the main coordinates system, then the output will produce the high resolution panorama image with a wide view angle.

However, some problem of stitching image will continually appear in multi image. Warped image with homography can be distorted view ${ }^{(6)}$. This problem can be avoided by converting image to cylindrical surface. For this method, lens (Focal length) is important to convert the normal image to the cylindrical image.

This paper has organized as following; Section 2 discusses how to stitch the regular panorama image, section 3 explains the cylindrical panorama, section 4 discusses how to stitching with cylindrical panorama, section 5 discusses the experimental result, and section 6 discusses the conclusion and direction for future work.

\section{Stitch regular panorama image}

Stitching image algorithm has been applied in many tasks such as capturing the overview of city, complex image form surface of the earth by satellites and image of travel location $^{(7)}$. The main deployment will focus on realistic results instead of effective results

\subsection{Image stitching Algorithm}

The stitching image algorithm is divided into 3 steps as shown in Fig. 1 


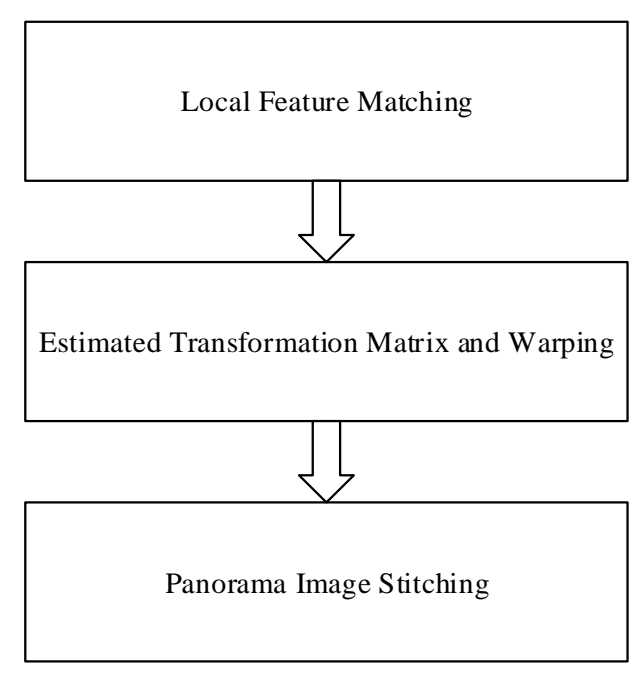

Fig. 1. Stitching image algorithm

\section{(a) Local Feature Matching}

The overlapping area position is extracted from the feature of image such as value of color, pixel density and etc. In this paper, SURF algorithm ${ }^{(8)}$ will used to extract the features, which is fast and powerful resistance to change of image such as rotation, scale, blur, and value of illuminance. SURF algorithm is detecting the feature from the second derivative of Gaussian and descripts using haar wavelet for integral image. That is less computational time. The output called key points is able to identify the location, scaling, and orientation as shown in Fig 2.

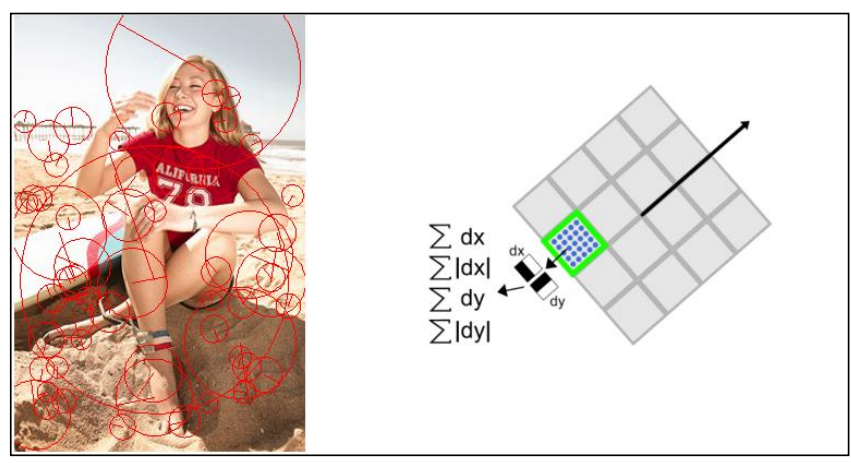

Fig. 2. Feature obtain SURF algorithm

Normally, the similarity between the images is calculated by the Euclidian distance. The small value of distance means that the two points is similar. In conversely, they are different. In this paper, the threshold of similarity is set to 0.6 as shown in Fig. 3

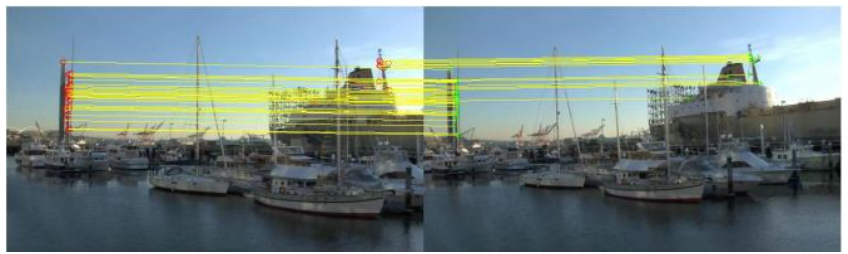

Fig. 3. Position of similar key points between 2 images (left) main image (right) reference image

Form Fig. 3, the overlapping area can be obtained from the position of matching key points. Form the left and right image, points of similarity will be matched with line and drawn between 2 images. After this process the overlapping area will show.

(b) Estimated transformation matrix and warping

This step is for transforming the images to the same view. Some overlapping image may be found the problems because of difference of view between images such as translation, rotation, scale, etc as shown in Fig. 4

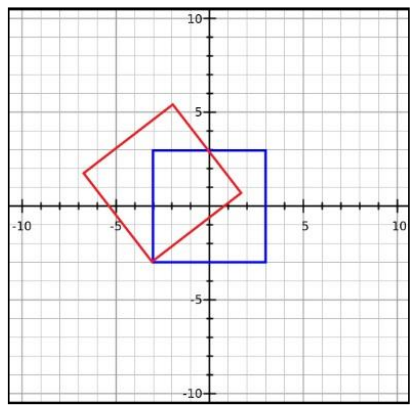

Rotation

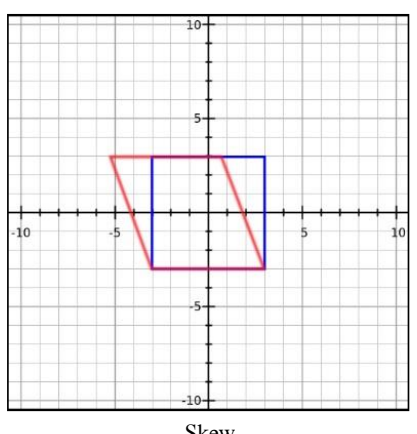

Fig. 4. Geometric transformation

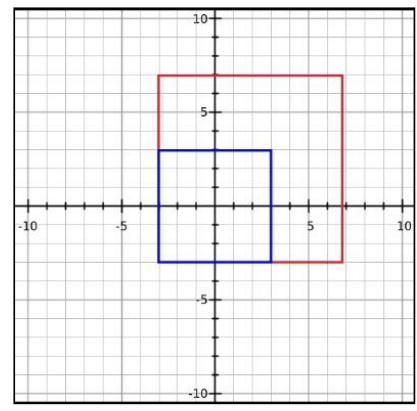

Scale

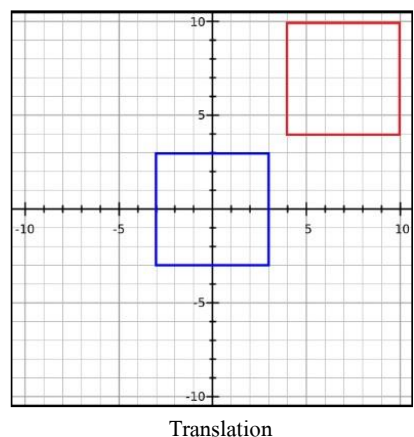

If the image are not transformed to the same view the result after stitch will distort, and it is uncontinue.

This problem can be solved by transformation of image to the same view, and use the corresponding points from the previous step for estimated transformation matrix. The wellknown estimated algorithm is the random sample consensus (RANSAC) $)^{(9)}$. Advantage of this method is the robust 
estimation of the homography matrix. RANSAC selectes or prunes the corresponding points by fitting the homography.

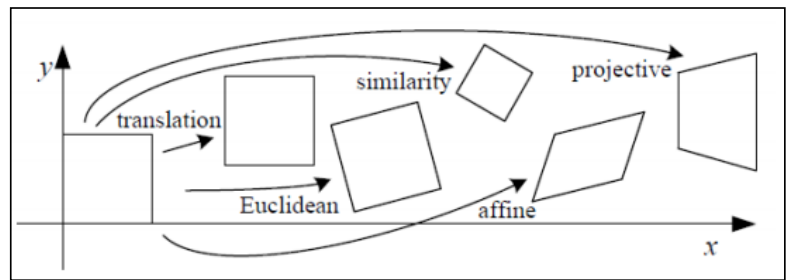

Fig. 5. Types of transformation function ${ }^{(10)}$

The transformation functions are various types as shown in Fig. 5. Each model has different properties such as Translation model can slide image to aspiring position, Euclidean model can increase rotation from Translation model, Similarity model can increase the scale from Euclidean model, Affine model can increase shearing from similarity work at same depth, and Projective model can transform all types of images. This paper selected to use the transformation function by Projective model, which uses the homography matrix $3 \times 3$ to transform and warping image calculated by equation (1).

$$
x_{2}=H x_{1} \text { given } x_{1}, x_{2} \in \mathbb{R}^{3}
$$

where $x_{2}$ is the result after warping, $x_{1}$ is image aspiring warping and $H$ is the $3 \times 3$ homography matrix. Homography matrix is shown in equation (2).

$$
\left[\begin{array}{l}
x_{2} \\
y_{2} \\
z_{2}
\end{array}\right]=\left[\begin{array}{lll}
H_{11} & H_{12} & H_{13} \\
H_{21} & H_{22} & H_{23} \\
H_{31} & H_{32} & H_{33}
\end{array}\right]\left[\begin{array}{l}
x_{1} \\
y_{1} \\
z_{1}
\end{array}\right]
$$

$\mathrm{H}$ is able to solve the equation using normalized direct linear transformation (DLT) ${ }^{(11)}$ which is computed by the corresponding points at least 4 pairs. The number of corresponding points effect with the error, and that are categorized into 2 types; inlier, and outlier by RANSAC classification. Inlier points used to compute the homography for warping image as shown in Fig. 6

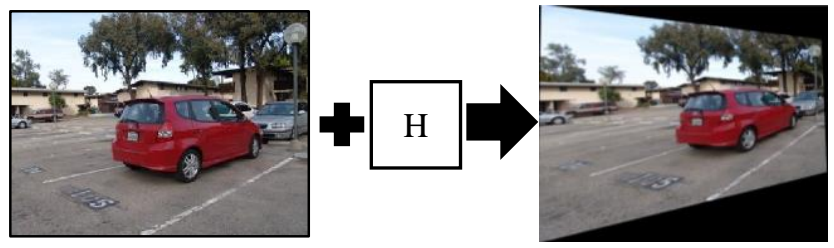

Fig. 6. Warping image result
The left image of Fig. 6 is the image before warping and the right image is the change of view of image after warping.

(c) Panorama image stitching

Warped image is used to create a panorama image. Each of position of image must correctly proper, using the data of homography. The result of image stitching is shown in Fig. 7

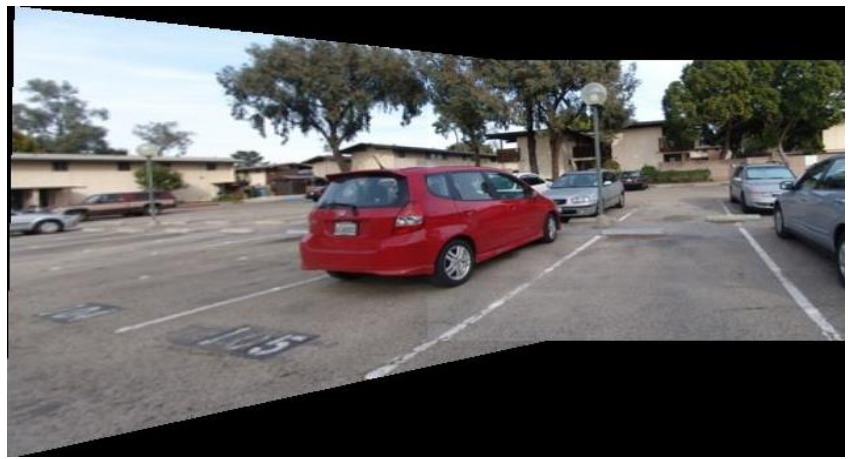

Fig. 7. Results after the end of the process, the panorama.

\subsection{Encountered problem in the regular panorama}

This problem is appearing on the warped image for stitching on many images. Example of distortion result is shown in Fig. 8

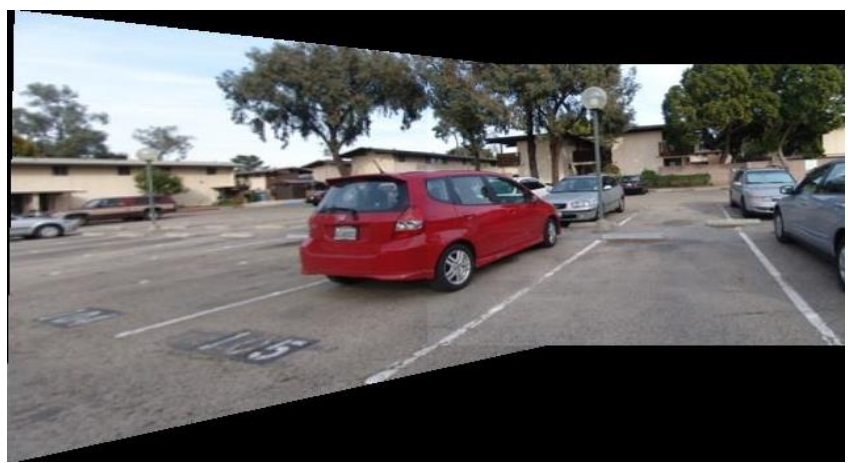

Fig. 8 Result Distortion Problem

Although, homography based method can refer to any type of images, but stitching on many image in one direction (landscape) will increase the distortion on image, caused by type of warping. The cross product of homography is used to stitch the image to the same view. However the problem is found from that. 


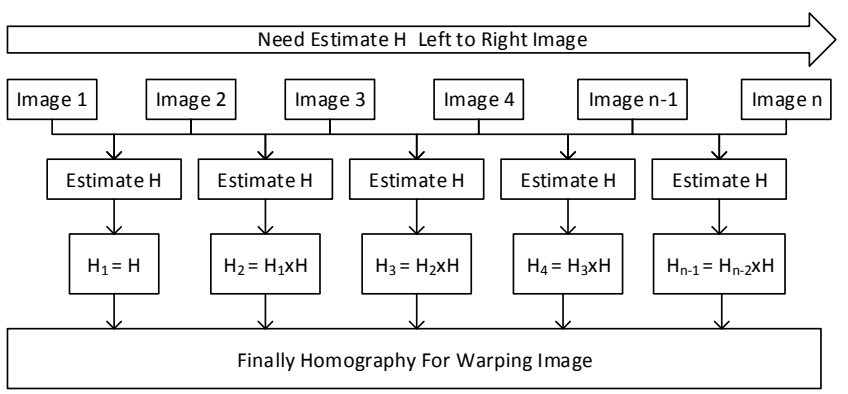

Fig. 9 Homography for warping image process

From Fig. 9, the panorama images must stitch the image from left to right. The number image is $n$. In this process, image 2 needs to transform to the same view of image 1 , and image 3 needs to transform to the same view of image 2 , and respectively. The number of homography matrix is $n-1$. From these homography matrix, the images are still not stitching. homography matrix must calculate the cross product such as $\mathrm{H}_{2}$ cross product with $\mathrm{H}_{1}$ with present $\mathrm{H}, \mathrm{H}_{3}$ cross product with $\mathrm{H}_{2}$ with present $\mathrm{H}$, respectively. The cross product will make for the cumulative value of translation matrix for correct position. In conversely, homography matrix produces the cumulative distort value, that the panorama result will distort, indefinitely. From Fig. 10, the position of T7 and T8 in the matrix are controlling the distorting value.

\begin{tabular}{|c|c|c|}
\hline T1 & T2 & T3 \\
\hline T4 & T5 & T6 \\
\hline T7 & T8 & T9 \\
\hline
\end{tabular}

Fig. 10 Distortion control matrix

Example distortion from T7 and T8 as shown in Fig. 11

\begin{tabular}{|l|l|l|}
\hline 1 & 0 & 0 \\
\hline 0 & 1 & 0 \\
\hline 0 & 0 & 1 \\
\hline
\end{tabular}

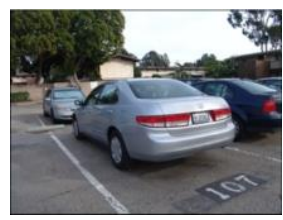

(a) Initial homography matrix

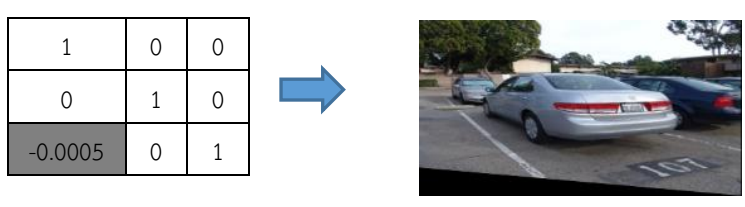

(b) Distortion of image 1

\begin{tabular}{|c|c|c|}
\hline 1 & 0 & 0 \\
\hline 0 & 1 & 0 \\
\hline-0.001 & $y$ & 1 \\
\hline
\end{tabular}

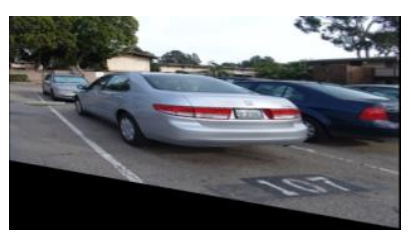

(c) Distortion of image 2

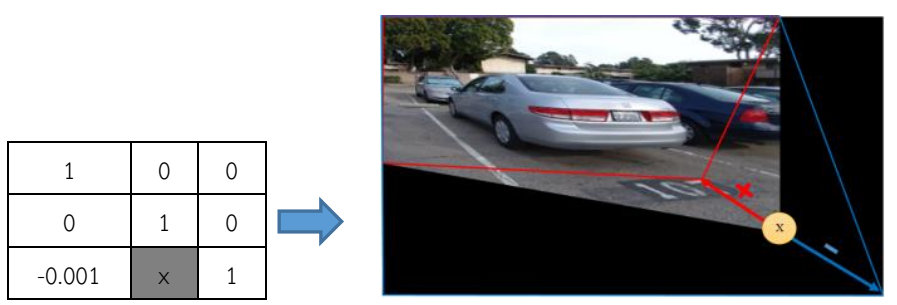

(d) Distortion of image 3

Fig. 11 The example of distortion matrix

Fig. 11 (a) shows the original image and initial homography matrix (identity matrix), (b) and (c) show the distortion image that the weight are equal (-0.0005 and $-0.001)$. (d) shows that the $\mathrm{x}$ is greater than 0 , the images is smaller than original, when $\mathrm{x}$ tends to 1 . In contrast, images is bigger than original when $\mathrm{x}$ tends to -1 . The value of $\mathrm{T}_{7}$ and $\mathrm{T}_{8}$ value will cumulate distortion in case of panorama.

This problem is able to solve by converting the images from rectangle to cylindrical projection, instead of homography matrix for estimating translation transform.

\section{Cylindrical Panorama}

Cylindrical image is different view from the original image, as shown in Fig. 12

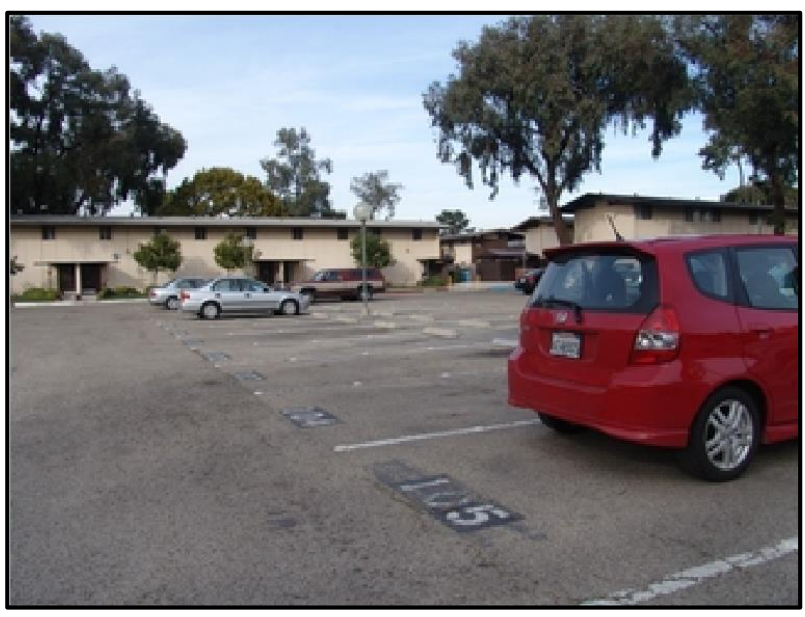

(a) Original image 


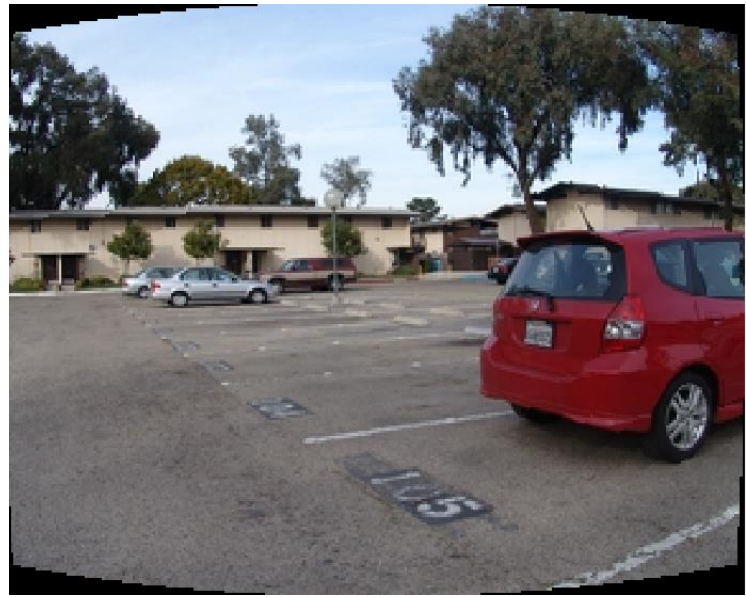

(b) Cylindrical image

Fig. 12 Original to cylindrical image

Original image are square shape but top and bottom of cylindrical image are curve. The advantage of cylindrical transformation for wide panorama stitching, that it is able to create the non-distorted panorama. The vital parameter of cylindrical transformation is focal length (distance from the focus point of the system to the surface and control field of view) to control projection of cylindrical image (curving). Cylindrical image is computed by equation (3) and (4).

$$
\begin{gathered}
x^{\prime}=f * \tan \left(\frac{x-x_{c}}{f}\right)+x_{c} \\
y^{\prime}=\frac{y-y_{c}}{\cos \left(\frac{x-x_{c}}{f}\right)}+y_{c}
\end{gathered}
$$

where $\left(x_{c}, y_{c}\right)$ is the old coordinate of $(x, y),\left(x^{\prime}, y^{\prime}\right)$ is the new coordinate of $(x, y), x$ is the width of image, $y$ is the height of image, and $\mathrm{f}$ is a focal length.

\section{Stitching with Cylindrical Panorama}

This chapter discusses to solve the panorama image stitching problem. The main objective is not distorting result. This process has similar to the regular image stitching, but it use using the translation transform instead of projective transform as shown in Fig. 13

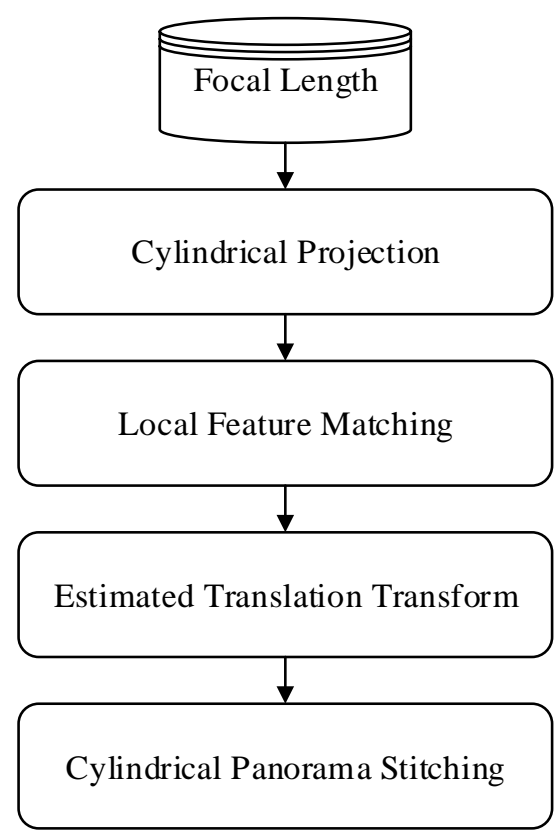

Fig. 13 Stitching cylindrical image algorithm

(a) Cylindrical Projection

Cylindrical projection is image transformation to cylindrical surface. The focal length used to stitch the images to the same perspective. The result are shown in Fig. 14
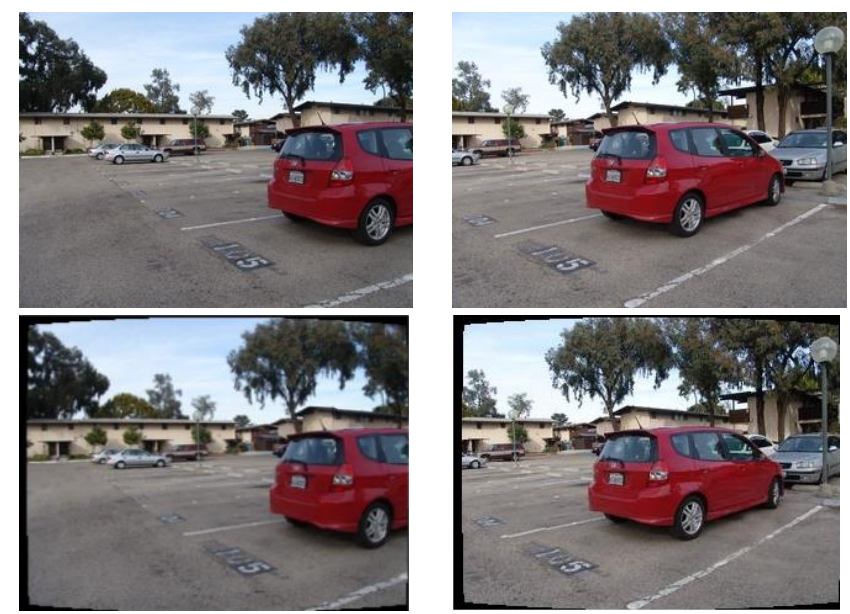

Fig. 14 Sample image cylindrical projection

\section{(b) Local Feature Matching}

This process is feature extraction from SURF algorithm, and using Euclidian distance to find the corresponding points as shown in Fig. 15 


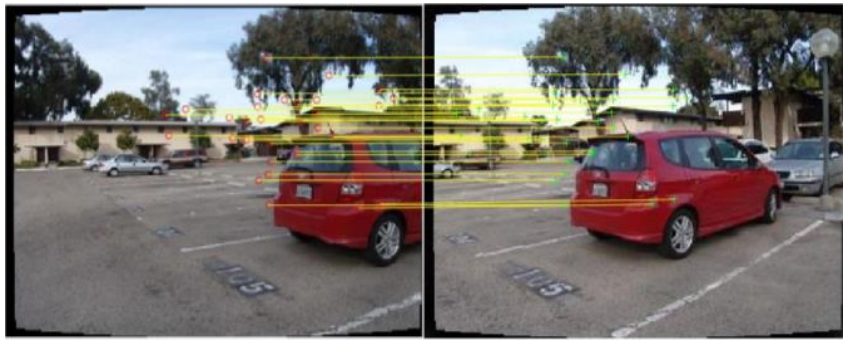

Fig. 15 Corresponding points from cylindrical images

(c) Estimated Translation Transform

This process uses translation matrix instead of homography matrix, and estimates translation transform by RANSAC. Translation matrix is shown in equation (5)

$$
\left[\begin{array}{l}
x_{2} \\
y_{2} \\
z_{2}
\end{array}\right]=\left[\begin{array}{ccc}
1 & 0 & T_{x} \\
0 & 1 & T_{y} \\
0 & 0 & 1
\end{array}\right]\left[\begin{array}{l}
x_{1} \\
y_{1} \\
z_{1}
\end{array}\right]
$$

where $\left(x_{1}, y_{1}, z_{1}\right)$ is old coordinates and $\left(x_{2}, y_{2}, z_{2}\right)$ is new coordinates after image transformation and $\left(\mathrm{T}_{\mathrm{x}}, \mathrm{T}_{\mathrm{y}}\right)$ is the control translation image value. One pair of corresponding points for estimated translation transform is $A\left(x_{1}, y_{1}\right)$ and $B\left(x_{2}, y_{2}\right)$ calculated by equation (6) and (7)

$$
\begin{aligned}
& T_{x}=x_{2}-x_{1} \\
& T_{y}=y_{2}-y_{1}
\end{aligned}
$$

where $T_{x}$ is the value of translation in $x$ axis, $T_{y}$ is the value of translation in $\mathrm{y}$ axis and $\left(x_{1}, y_{1}, x_{2}, y_{2}\right)$ is position coordinate system. Result is shown in Fig. 16.

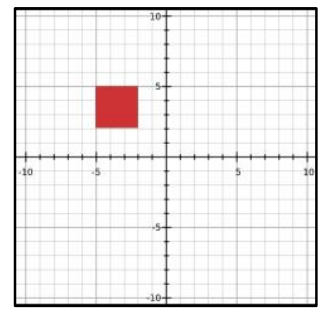

(a) Before translation

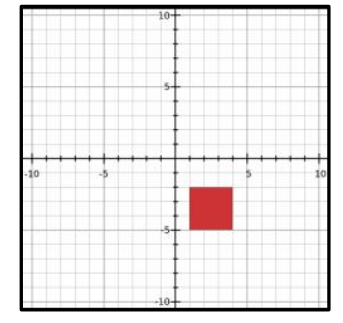

(b) After translation
Fig. 16 Translation Transform

From Fig. 16 (a) the $T_{x}$ and $T_{y}$ are equal to 6 and -7 , will move to the new coordinate as shown in Fig. 16 (b)

(d) Cylindrical panorama stitching

In this paper, image stitching is performed begin from left to right and first to last of image. The result is shown in Fig. 17

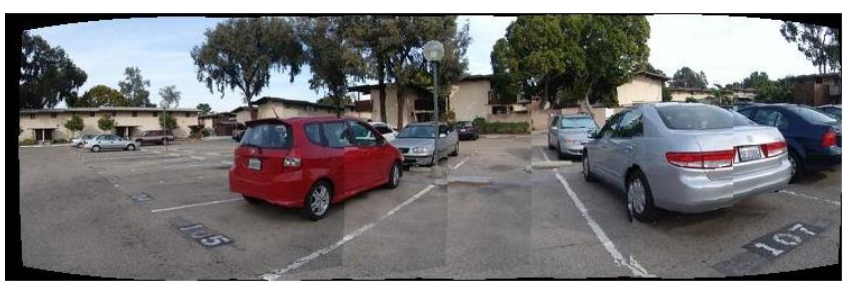

Fig. 17 Example cylindrical panorama

\section{Experiment and Result}

The experimental result is divided into 2 parts; perspective view and computational time. The data collection is came from 2 sources that are internet and normal camera. The number of image datasets collected on the internet are 10 sets came from "www.visualsize.com/Mosaic3D”. The number of normal camera image are 5 sets. All images are resize to 480x320 pixels. Example image datasets are shown in Fig. 18.
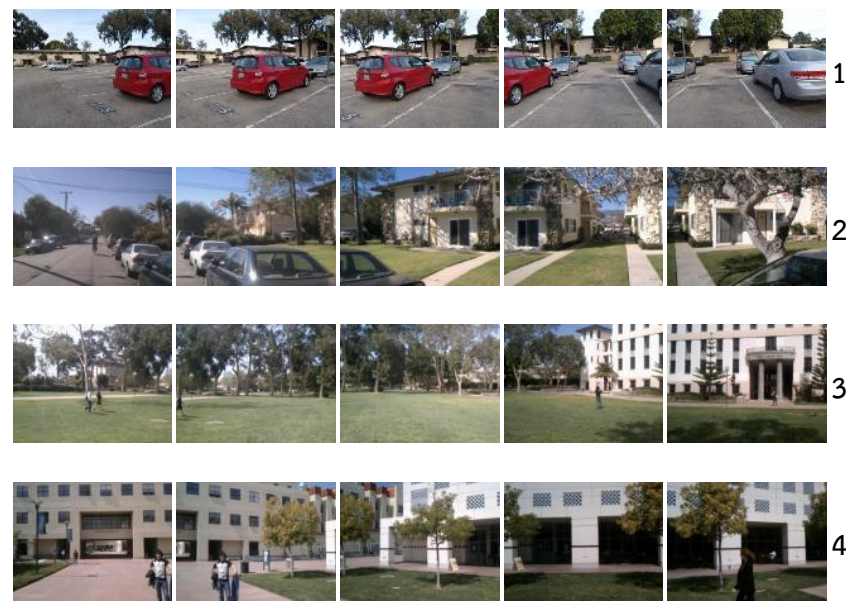

Fig. 18 Example datasets

Normally, image stitching is excluding the image blending and the optimal seam for smooth images. The comparison between regular stitching image and cylindrical stitching image for various number of images are shown in Fig. 19 and Fig. 20
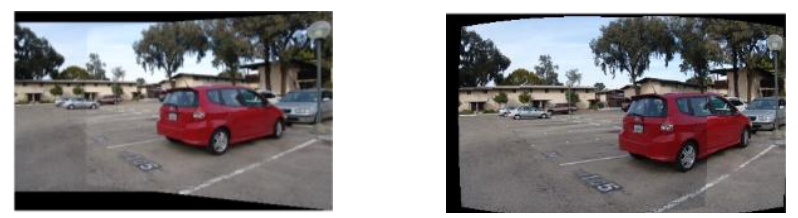

(a) Number of 2 Image regular panorama (left) cylindrical panorama (right) 

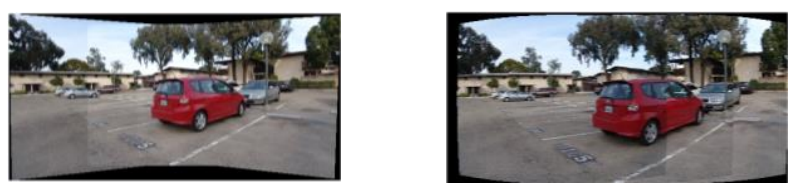

(b) Number of 3 Image regular panorama (left) cylindrical panorama (right)
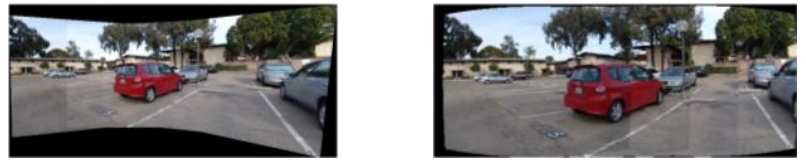

(c) Number of 4 Image regular panorama (left) cylindrical panorama (right)
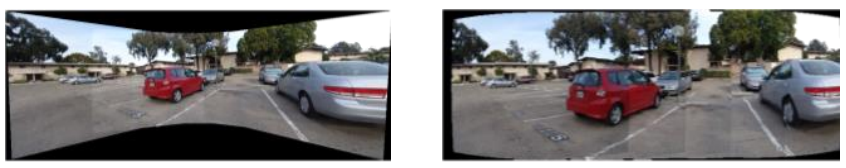

(d) Number of 5 Image regular panorama (left) cylindrical panorama (right)

Fig. 19. Example Stitching Panorama Dataset 1 of various number of images
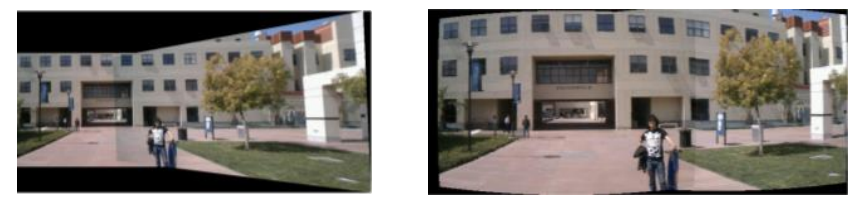

(a) Number of 2 Image regular panorama (left) cylindrical panorama (right)
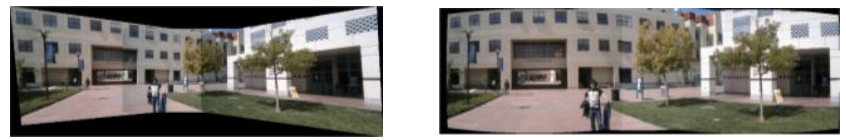

(b) Number of 3 Image regular panorama (left) cylindrical panorama (right)
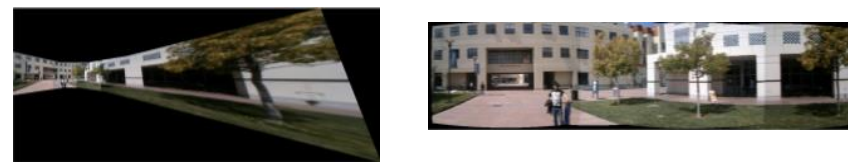

(c) Number of 4 Image regular panorama (left) cylindrical panorama (right)
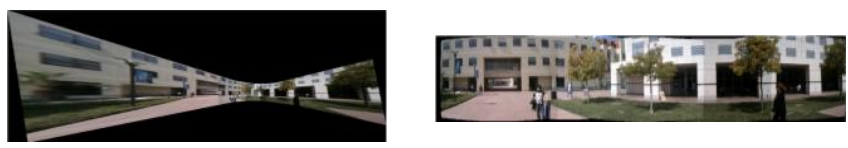

(d) Number of 5 Image regular panorama (left) cylindrical panorama (right)

Fig. 20. Example Stitching Panorama Dataset 2 of various number of images

From Fig. 19 (a) and Fig. 20 (a), the both panoramas result from stitching between 2 images are the same. That are in same perspective and still no distortion. For Panorama stitching of 3 images from (Fig. 19 (b), Fig. 20 (b)), regular stitching panoramas begin little distortion. However, Panorama stitching of 4 and 5 images (Fig. 19 (c), (d), Fig. 20 (c), (d)), regular stitching panoramas appear explicit distortion but cylindrical stitching panoramas are still no distortion.
In generally, the regular panorama stitching for 2 or 3 images performs faster than cylindrical panorama stitching because of projection process. However, in case of 4 image up the cylindrical panorama stitching performs faster than regular panorama stitching as shown in Table 1.

Table 1. Time experiment

\begin{tabular}{|c|c|c|c|c|}
\hline & \multicolumn{4}{|c|}{ Mean time (second) } \\
\hline $\begin{array}{c}\text { Image } \\
\text { number }\end{array}$ & 2 & 3 & 4 & 5 \\
\hline Regular & 0.21 & 0.4 & 8.99 & 23.01 \\
\hline Cylindrical & 1.54 & 2.51 & 3.59 & 4.75 \\
\hline
\end{tabular}

\section{Conclusion}

Normally, wide panorama stitching using regular panorama stitching are distortion. Most of distortion occur from horizontal image of four or more images. Moreover, the stitching image process is failure. Experimental results show that the cylindrical panorama stitching successfully solves these problems.

The output image still found the unsmooth, thus the future work will propose the blending algorithm and try to increase the efficiency of stitching by automatic estimated focal length.

\section{Acknowledgment}

The research is supported by the Thailand Government funding from Mahasarakham University, 2015.

\section{References}

(1) Quan-hai Wang, Fang Miao, Biao Lie, and Can-Ping Li : "Panorama Image Distortion Correction Method based on Straight Baseline", Apperceiving Computing and Intelligence Analysis, Vol. , No. ,pp. 203-207, 2008

(2) Saeed Salehi and Eric Dubois : "Alignment of CubicPanorama Image Datasets using Epipolar Geometry International Conference on Acoustics", Signal Processing (ICASSP), Val. , No. , pp. 1545-1548, 2011

(3) Tao-Cheng Chang, Cheng-An Chien, Jia-Hou Chang, and Jiun-In Guo : "A Low-Complexity Image Stitching Algorithm Suitable for Embedded Systems", International Conference on Consumer Electronics (ICCE), Vol. , No. , pp. 197-198, 2011 
(4) Yingen Xiong and Kari Pulli : "Sequential image stitching for mobile panoramas", Information Communications and Signal Processing, Vol. , No. , pp.1-5, 2009

(5) Hongming Zhang, Wei Zeng, and Xin Chen : "Foreground Based Borderline Adjusting for Real Time Multi-camera Video Stitching", Image and Graphics (ICIG'09), Vol. , No. , pp.335-360, 2009

(6) Geetha Kiran, and Murali S : "Automatic rectification of perspective distortion from a single image using plane homography", Vol.3, No.5, pp. 47-58, 2013

(7) Jubiao Li and Junping Du : "Study on panoramic image stitching algorithm", Communications and System (PACCS), Vol. 1, No. , pp. 417-420, 2010

(8) Herbert Bay, Andreas Ess, Tinne Tuytelaars, and Luc Van Gool : "Surf: Speeded up robust features", Computer vision and image understanding, Vol. 110, No. 3, pp. 346-359, 2008

(9) Martin A Fischler, and Robert C Bolles : "Random sample consensus: a paradigm for model fitting with applications to image analysis and automated cartography", Vol. 24, No. 6, pp. 381-395, 1981

(10) Richard Szeliski : "Image alignment and stitching : A Tutorial", Foundations and Trends ${ }^{\circledR}$ in Computer Graphics and Vision, Vol. 2, No. 1, pp. 1-104, 2006

(11) YI Abdel-Aziz and HM Karara : "Direct linear transformation from comparator coordinates into object space coordinates in close-range photogrammetry", ASP Symposium on Close-Range Photogrammetry in Illinois, Vol. , No. , pp. 1-18, 1971 